\title{
Flora da Bahia: Hypoxidaceae
}

\author{
Karoline Coutinho de Santana $^{1^{*}}$, Reyjane Patricia de Oliveira ${ }^{1, a}$ \& Ana Maria Giulietti ${ }^{1,2, b}$ \\ ${ }^{1}$ Programa de Pós-Graduação em Botânica, Departamento de Ciências Biológicas, Universidade Estadual de \\ Feira de Santana, Feira de Santana, Bahia, Brasil. \\ 2 Instituto Tecnológico Vale, Belém, Pará, Brasil.
}

Resumo - É apresentado aqui o tratamento taxonômico das Hypoxidaceae da Bahia, Brasil. Hypoxis decumbens é única espécie encontrada no Estado. A Flora consiste de descrições, ilustrações, comentários e um mapa de distribuição geográfica da espécie na Bahia.

Palavras-chave adicionais: Brasil, florística, Hypoxis, monocotiledôneas, Nordeste.

\begin{abstract}
Flora of Bahia: Hypoxidaceae) - The taxonomic treatment of the Hypoxidaceae from Bahia, Brazil, is presented here. Hypoxis decumbens is the only species found in the state. Descriptions, illustrations, comments and a geographic distribution map of the species in Bahia are presented.
\end{abstract}

Additional key words: Brazil, floristics, Hypoxis, monocots, Northeast Brazil.

\section{HYPOXIDACEAE}

Ervas terrestres, perenes, com cormo (Hypoxis L.) ou rizoma (Curculigo Gaertn.). Folhas basais, simples, alternas trísticas, com nervuras paralelas proeminentes, frequentemente plicadas, sésseis ou pseudopecioladas, pilosas a glabescentes. Inflorescências axilares, cimosas, espiciformes a corimbosas ou umbeliformes, às vezes reduzidas a uma única flor; escapos geralmente pilosos. Flores vistosas, amarelas a alaranjadas, raramente vermelhas, pediceladas ou sésseis, bissexuadas ou unissexuadas (Curculigo), actinomorfas, diclamídeas, homoclamídeas; tépalas (3)6, livres, persistentes; estames (3)6, livres ou basalmente adnatos às tépalas, opostos às tépalas internas; anteras basifixas ou dorsifixas, rimosas; ovário ínfero, 3-carpelar, (1)3locular, cilíndrico, pluriovulado, placentação axilar ou raramente parietal. Frutos bagas (Curculigo) ou cápsulas (Hypoxis), deiscência circuncisa ou loculicida, às vezes indeiscente ou irregularmente deiscente; sementes globosas ou elipsoides, lisas, rostradas, às vezes verrucosas.

Hypoxidaceae pertence à ordem Asparagales (APG IV 2016) e agrupa cerca de 200 espécies (Liu et al. 2012). A delimitação de seus gêneros é controversa, baseada em poucos caracteres que se sobrepõem. Como consequência, o número de gêneros ainda é incerto; alguns autores (e.g., Nordal 1998) consideram nove, outros apenas sete (e.g., Govaerts 2016). A família está distribuída predominantemente nas regiões tropicais e subtropicais da África, Ásia, Austrália e Américas, com a maioria das espécies concentradas no hemisfério sul (Nodal 1998). Na Região Neotropical, ocorrem apenas dois gêneros (Dulith 2009), Hypoxis e Curculigo, ambos encontrados no Brasil (Dulith 2016).

\footnotetext{
*Autora para correspondência: karolinecoutinho@yahoo.com.br; arpatricia@uefs.br; banagiulietti@hotmail.com

Editor responsável: Alessandro Rapini

Submetido: 31 maio 2016; aceito: 25 out. 2016

Publicação eletrônica: 1 nov. 2016; versão final: 7 nov. 2016
}

Hypoxis decumbens L. e C. scorzonerifolia (Lam.) Baker foram referidas para a Bahia (Dulith 2016); contudo, nenhuma amostra de Curculigo foi localizada em campo ou nos herbários consultados (ALCB, HUEFS, CEPEC, HRB e HURB).

\section{Hypoxis L.}

Ervas de pequeno a médio porte; cormo globoso, alongado ou elipsoide. Folhas sésseis, eretas ou ascendentes, lineares a lanceoladas, em geral pilosas, às vezes decompondo-se em fibras persistentes na base. Inflorescências cimosas ou racemosas; escapos comumente filiformes, ascendentes, pilosos; brácteas quando presentes subuladas a lineares. Flores pediceladas a sésseis; tépalas amarelas, pilosas na face externa, persistentes; estames 6 , filetes inseridos na base das tépalas, anteras basifixas, levemente sagitadas, deiscência rimosa; ovário trilocular cilíndrico, piloso. Frutos cápsulas circuncisas ou deiscentes longitudinalmente, subglobosas a subcilíndricas, geralmente trilobadas.

Hypoxis é o maior gênero de Hypoxidaceae, com cerca de 90 espécies, distribuídas nas Américas, África, Ásia e Austrália (Singh 2007). Podem ser encontradas em diferentes hábitats, desde áreas encharcadas até áreas de solo rochoso e com pouca disponibilidade hídrica (Nordal 1998). É facilmente caracterizado pelo caule do tipo cormo, folhas com tricomas simples e inflorescência cimosas ou racemosas, enquanto Curculigo possui caule rizomatoso, folhas com tricomas bifurcados ou raramente estrelados e flores solitárias ou reunidas em umbelas (Zimudzi 1994). A delimitação das espécies em Hypoxis, por outro lado, é problemática, o que geralmente é atribuído à ocorrência de hibridações e/ou apomixia em grande parte das espécies (Sklenar 2005).

Hypoxis decumbens L., Syst. Nat. (ed. 10) 2: 986. 1759. Figuras 1-3.

Ervas 8-30 cm alt; cormo 0,4-1,1 cm diâm, tuberoso, fibroso, cilíndrico. Folhas $6-48 \times 0,3-1 \mathrm{~cm}$, 
ascendentes, membranáceas, lineares a lanceoladas, ápice agudo, margem inteira, pilosas a glabescentes. Inflorescências axilares, 1-5-floras; escapo 4-21 cm compr., filiforme, tricomas esbranquiçados ou amarelos; brácteas $0,5-1 \mathrm{~cm}$ compr., filiformes a lanceoladas, ápice agudo; botões florais $0,6-0,8 \times 0,35-0,45 \mathrm{~cm}$, hirsuto-pilosos. Flores 0,6-1,3 cm diâm.; pedicelo 0,1$0,4 \mathrm{~cm}$ compr., delgado, piloso; tépalas $0,4-0,6 \times 0,1-$ $0,3 \mathrm{~cm}$, amarelas, elípticas a lanceoladas, ápice agudo, as externas densamente pilosas abaxialmente, fortemente enervadas, as internas glabras ou pouco pilosas; estames livres, anteras $0,1-0,15 \mathrm{~cm}$ compr., glabras, bitecas, dorsifixas, filetes $0,15-0,2 \mathrm{~cm}$ compr., filiformes, glabros; ovário $0,2-0,7 \mathrm{~cm}$ compr., cilíndrico, hipanto piloso, estilete $0,2-0,3 \mathrm{~cm}$. Cápsulas 0,7-2 cm compr., cilíndricas a elipsoides, geralmente pilosas, com deiscência circuncisa; sementes $0,08-0,1 \times$ $0,08-0,1 \mathrm{~cm}$, globosas a subglobosas.

Ocorre nas Américas, Antilhas, África, leste e sudeste da Ásia e Austrália (Grayum 2003), geralmente em ambientes ruderais, campos abertos, pastos e locais úmidos (Pena et al. 2008); no Brasil, ocorre em todo o território (Dutilh 2016), em áreas antropizadas. C7, E6, E8, E9, F6, F7, F8, G8, I8: caatinga, campo rupestre, cerrado, mata atlântica e restinga. A floração e frutificação ocorrem de fevereiro a setembro.

Material selecionado: Amargosa, fazenda Timbó, 13ำ's, 3909’W, 13 mar. 2007 (bot., fl.), J.L. Paixão et al. 1120 (HUEFS); Amélia Rodrigues, 12²6'29”S, 3844'02”W, 20 mar. 1987 (fl., fr.), L.P. Queiroz \& I.C. Crepaldi 1466 (HUEFS); Cachoeira, mata a nordeste da barragem Bananeiras, jul. 1980 (fr.), Grupo Pedra do Cavalo 426 (ALCB); Cascavel, entre Lagoa Encantada e Cascavel, 13¹8'34'S, 4150'12'W, 21 mar. 1999 (fr.), R.M. Harley et al. 53570 (HUEFS); Cruz das Almas, 3 mar. 1981 (fr.), G.C.P. Pinto s.n. (HRB 2158); Entre Rios, Imbé, $12^{\circ} 03^{\prime} \mathrm{S}, 38^{\circ} 02^{\prime} \mathrm{W}, 13$ ago. 2010 (fr.), A.V. Popovkin \& Mendes 729 (HUEFS); Feira de Santana, Jaíba, 12¹3'53”S, 38 52'29”'W, 231 m s.n.m., 11 maio 2010 (fl., fr.), $M$. Silva et al. 32 (HUEFS); Ilhéus, Campus da Universidade Estadual de Santa Cruz, $14^{\circ} 48^{\prime}$ S, $30^{\circ} 10^{\prime} \mathrm{W}, 40$ m s.n.m. (bot., fr.), 8 set. 1995, L.A. Mattos Silva et al. 3188 (CEPEC); Itacaré, beira mar, 15 abr. 1970 (fr.), T.S. Santos 708 (CEPEC); Jaguaripe 1312'13”S, 3854'20”W, 13 jan. 2016 (fl.) G. Costa \& P.L. Ribeiro 1781 (HURB); Maracás, fazenda Vale Aprazível, 1329'06”S, 40²5'26”W, 1020 m s.n.m., 22 abr. 2002 (bot., fl., fr.) K.R.B. Leite et al. 197 (HUEFS); Mata de São

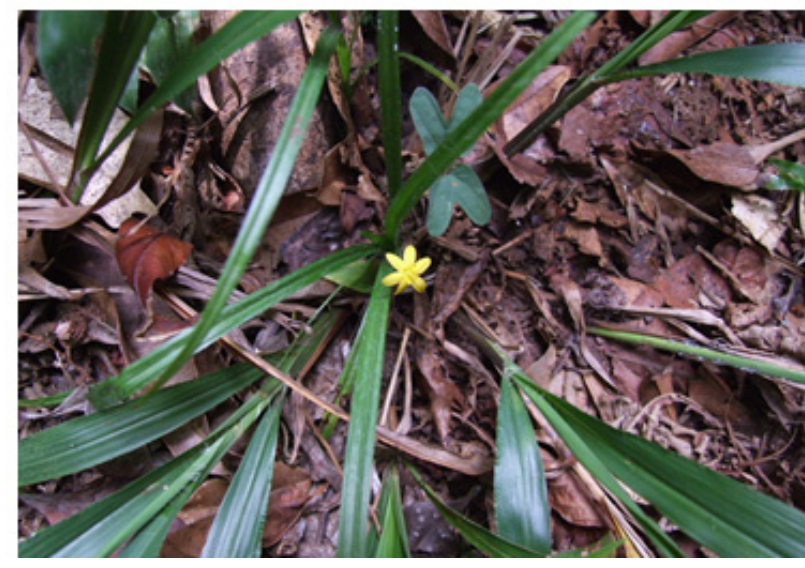

Figura 1. Hypoxis decumbens: planta com flor (Foto: D. Cardoso).

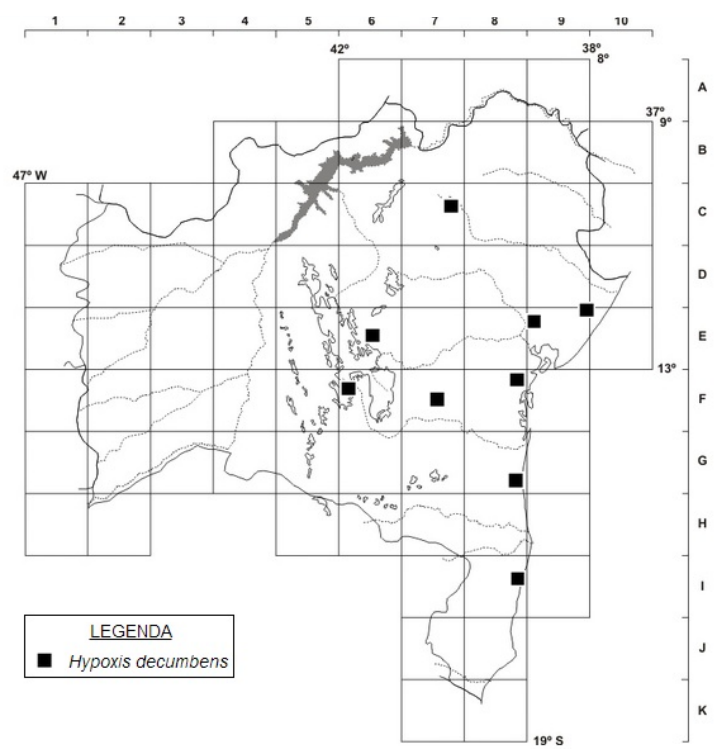

Figura 2. Distribuição geográfica de Hypoxis decumbens no estado da Bahia.

João, área da Caraíba Metais, 12²9’35”S 38¹8’47”W, 17 fev. 1983 (fr.), L.R. Nobick et al. 2552 (CEPEC); Morro do Chapéu, Ventura, 4 abr. 1997(fr.) P. Gasson PND 6000 (ALCB, CEPEC, HRB); Palmeiras, Serra dos Brejões, $12^{\circ} 27^{\prime} \mathrm{S}, 41^{\circ} 21^{\prime} \mathrm{W}, 1050 \mathrm{~m}$ s.n.m., 17 jul. 2008 (fl.), A.A. Conceição 2971 (HUEFS); Porto Seguro, rodovia BR-367, 13 maio 1980 (fl.), A. Eupunino 520 (CEPEC); Salvador, Horto Florestal, 1258'S, 38³0'W, 5 maio 1996 (fl., fr.), C.M. Longa \& G.L. Campos 5 (HRB); Santa Cruz Cabrália, Estação Ecológica de Pau-Brasil, 29 ago. 1983 (fr.), F.S. Santos 60 (CEPEC); Santa Teresinha, Serra da Jiboia, 1258’S, 38³0'W, maio 1999 (fr.) M.M. Silva et al. 222 (HUEFS); São Sebastião do Passé, Estação Experimental SOS Thenes Miranda, 12³0'45”S, 38²9'43”'W, 16 jul. 1983 (fr.), J.L. Hage et al. 1819 (CEPEC); Senhor do Bonfim, Povoado de Estiva, 10²1'57'S, 40¹1'51'W, 686 m s.n.m., 13 jul. 2005 (fl., fr), D. Cardoso et al. 704 (HUEFS).

Hypoxis decumbens é a única espécie do gênero referida para o Brasil, facilmente reconhecida pelas folhas ascendentes, lineares a lanceoladas, pilosas a glabrescentes, com flores pequenas $(0,6-1,3 \mathrm{~cm}$ diâm.), amareladas e pilosas. Apresenta ampla variação em relação à altura das plantas $(8-30 \mathrm{~cm})$, o que pode estar relacionado aos hábitats nos quais é encontrada. É referida como ruderal, sendo bem comum em todo o Brasil e apresenta distribuição pantropical.

\section{AgRADECIMENTOS}

À Universidade Estadual de Feira de Santana (UEFS) pela infraestrutura disponibilizada para a execução deste trabalho; aos curadores dos herbários pelo acesso às coleções; à Karena Pimenta pela ilustração; ao CNPq pelas bolsas de produtividade concedidas a AMG e RPO (PQ Senior e PQ-1D, respectivamente). Ao $\mathrm{CNPq}$ e à FAPESB pelo financiamento do Projeto Flora da Bahia (FAPESB APR 162/2007; CNPq processos 562278/2010-9 e 483909/2012-2) e do programa PPBIO Semiárido. 


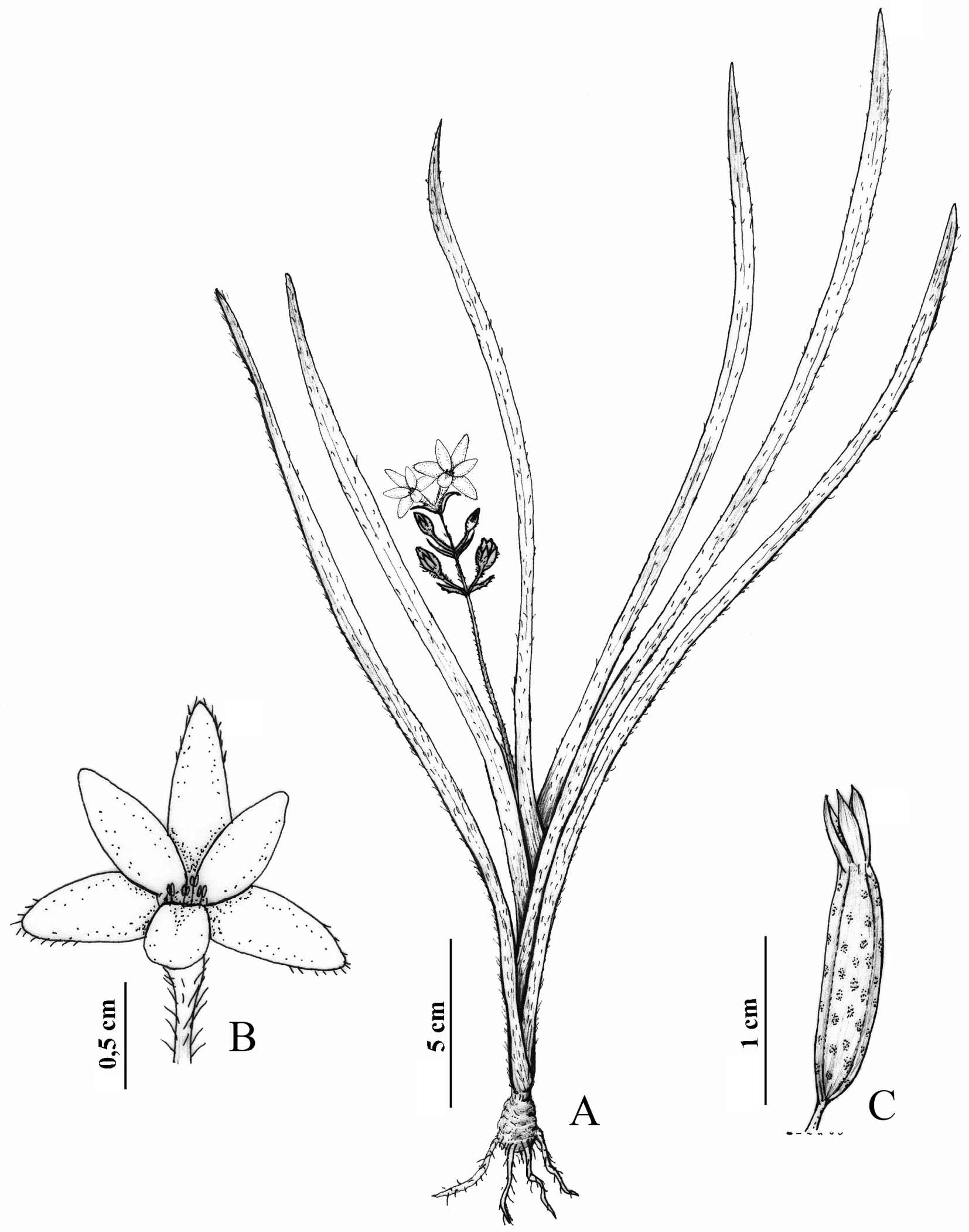

Figura 3. Hypoxis decumbens: A- hábito; B- flor; C- fruto (Rodal 1332).

\section{REFERÊNCIAS}

APG IV 2016. An update of the Angiosperm Phylogeny Group classification for the orders and families of flowering plants: APG IV. Botanical Journal of the Linnean Society 181: 1-20.
Dutilh, J.H.A. 2009. Neotropical Hypoxidaceae. In: W. Milliken, B. Klitgård \& A. Baracat, (2009 onwards), Neotropikey Interactive key and information resources for flowering plants of the Neotropics. http://www.kew.org/science/tropamerica/ neotropikey/families/Hypoxidaceae.htm. 
Dutilh, J.H.A. 2016. Hypoxidaceae. In: Lista de Espécies da Flora do Brasil. Jardim Botânico do Rio de Janeiro. Disponível em http://floradobrasil.jbrj.gov.br/jabot/floradobrasil/FB8021. Acesso em 18 jan. 2016.

Govaerts, R. 2016. World Checklist of Hypoxidaceae. Facilitated by the Royal Botanic Gardens, Kew. Disponível em http://apps.kew.org/wcsp; acesso em 31 maio 2016.

Grayum, M.H. 2003. Hypoxidaceae. In: B.E. Hammel, M.H. Grayum, C. Herrera \& N. Zamora (eds), Manual de plantas de Costa Rica. Vol. 2. Monographs in Systematic Botany from the Missouri Botanical Garden 92: 600-602.

Liu, K.-W.; Xie, G.-C.; Chen, L.-J.; Xiao, X.-J.;Zheng, Y.Y.;Cai, J.; Zhai, J.-W.; Zhang, G.-Q. \& Liu, Z.-J. 2012. Sinocurculigo, a new genus of Hypoxidaceae from China based on molecular and morphological evidence. PLoS ONE 7(6): e38880. doi:10.1371/journal.pone.0038880.
Nordal, I. 1998. Hypoxidaceae. In: K. Kubitzki (ed.), The Families and Genera of Vascular Plants. III. Flowering Plants. Monocotyledons. Lilianae (except Orchidaceae). SpringerVerlag, Berlin, p. 286-294.

Pena, M.A.; Watanabe, M.T.C. \& Sano, P.T. 2008. Flora da Serra do Cipó, Minas Gerais: Hypoxidaceae. Boletim de Botânica da Universidade de São Paulo 26(2): 61-68.

Singh, Y. 2007. Hypoxis (Hypoxidaceae) in southern Africa: taxonomic notes. South African Journal of Botany 77: 360-365.

Sklenar, P. 2005. Hypoxidaceae. In: P. Sklenar, J.L. Luteyn, C.U. Ulloa, P.M. Jorgensen \& M.O. Dillon (eds), Flora Genérica de los Páramos. Guía ilustrada de las plantas vasculares. The New York Botanical Garden, New York, p. 288-289.

Zimudzi, C. 1994. Revision of the genus Curculigo (Hypoxidaceae) in the Flora Zambesiaca area. Nordic Journal of Botany 14(3): 311-314.

\section{LISTA DE EXSICATAS}

Cardoso, D. 704; Conceição, A.A. 2971; Costa, G. 1781; Gasson, P. PCD 600; Grupo Pedra do Cavalo 327, 426; Guedes M.L. 37, 11799, 12713, 15085,19306; Hage, J.L. 1794; Harley, R.M. 22216, 53570; Leite, K.R.B. 197; Longa, C.M. 5; Mattos Silva L.A. 1737, 3188, 3596; Noblick, L.R. 2552, Nunes, T.S. 1242; Paixão, J.L. 1120; Pinto, G.C. s.n. HRB 2158; Popovkin, A.V. 729; Prates, A.R.S. 37 , 132, 179; Queiroz, L.P 1466; Rodal, M.J.N. 1332; Santos, F.S. 60; Santos, T.S 708; Silva, M.M 222. 